\title{
Stern-Gerlach Entanglement in Spinor Bose-Einstein Condensates.
}

\author{
S. Cruz-Barrios ${ }^{(1)}$, M. C. Nemes ${ }^{\left(2,3^{*}\right)}$ and A. F. R. de Toledo Piza ${ }^{(2)}$
}

October 31, 2018

\author{
(1) Departamento de Física Atómica, Molecular y Nuclear, Aptdo 1065, 41080 Sevilla \\ and Departamento de Física Aplicada I, Universidad de Sevilla \\ Sevilla, Spain \\ (2) Departamento de Física-Matemática, Instituto de Física, Universidade de São Paulo, \\ C.P. 66318, 05315-970 São Paulo, S.P., Brazil \\ (3) Departamento de Física, ICEX, Universidade Federal de Minas Gerais, \\ C.P. 702, 30161-970 Belo Horizonte, M.G., Brazil
}

\begin{abstract}
Entanglement of spin and position variables produced by spatially inhomogeneous magnetic fields of Stern-Gerlach type acting on spinor Bose-Einstein condensates may lead to interference effects at the level of one-boson densities. A model is worked out for these effects which is amenable to analytical calculation for gaussian shaped condensates. The resulting interference effects are sensitive to the spin polarization properties of the condensate.
\end{abstract}

PACS number: 03.75.Fi

The classic Stern-Gerlach experiment[1] is currently understood as providing for the spin analysis of a beam of particles using the entanglement of spin and spatial degrees of freedom through the action of inhomogeneous magnetic fields and subsequent spatial analysis of the scattered flux [2]. In this paper we explore effects resulting from this same type of entanglement when an essentially static spinor Bose-Einstein condensate with large coherence length is subjected to Stern-Gerlach field gradients. In the case of pure condensates that are sufficiently dilute so that mean field effects are a minor correction, the involved dynamics can be treated in a rather straightforward and simple way for appropriate, non-trivial field configurations using the ideas recently developed in ref. [3].

A simple but still rather general model state for a pure spinor (spin 1 for definiteness) condensate is one in which all bosons occupy one same single-boson spin-orbital $u(\vec{r}, \sigma)$,

\footnotetext{
${ }^{*}$ Permanent Address.
} 
where $\sigma=-1,0,1$ is the spin variable. This state can be expressed in terms of the creation operator

$$
a^{\dagger} \equiv \sum_{\sigma} \int d^{3} r u(\vec{r}, \sigma) \psi_{\sigma}^{\dagger}(\vec{r})
$$

where $\psi_{\sigma}(\vec{r})$ is the spinor bosonic field operator, as

$$
\left|\Psi^{(N)}\right\rangle=\frac{1}{\sqrt{N !}}\left(a^{\dagger}\right)^{N}|0\rangle
$$

This state is completely characterized by its one-boson density, given by

$$
\rho\left(\vec{r}, \sigma ; \vec{r}^{\prime} \sigma^{\prime}\right) \equiv\left\langle\Psi^{(N)}\left|\psi_{\sigma^{\prime}}^{\dagger}\left(\vec{r}^{\prime}\right) \psi_{\sigma}(\vec{r})\right| \Psi^{(N)}\right\rangle=N u(\vec{r}, \sigma) u^{*}\left(\vec{r}^{\prime}, \sigma^{\prime}\right) .
$$

The spin and position degrees of freedom are entangled whenever the spin-orbital $u(\vec{r}, \sigma)$ does not have the product form $u(\vec{r}) \chi(\sigma)$. A slightly more general form for a non-entangled one-body density is

$$
\rho\left(\vec{r}, \sigma ; \vec{r}^{\prime} \sigma^{\prime}\right) \rightarrow u(\vec{r}) u^{*}\left(\vec{r}^{\prime}\right) \otimes \rho^{(\mathrm{s})}\left(\sigma ; \sigma^{\prime}\right) \equiv \rho^{(\mathrm{pos})}\left(\vec{r} ; \vec{r}^{\prime}\right) \otimes \rho^{(\mathrm{s})}\left(\sigma ; \sigma^{\prime}\right)
$$

where the second factor, representing the spin density matrix, is allowed to be a mixed state. This information if of course not sufficient to craracterize the underlying many-boson state. Considering, as an example, a case in which $\rho^{(\mathrm{s})}\left(\sigma ; \sigma^{\prime}\right)$ is diagonal, with eigenvalues $N_{\sigma}$ and $\sum_{\sigma=-1}^{1} N_{\sigma}=N$, both the pure $N$-boson state

$$
\left|\left\{N_{\sigma}\right\}\right\rangle=\frac{1}{\sqrt{N_{1} ! N_{0} ! N_{-1} !}} \prod_{\sigma}\left(a_{\sigma}^{\dagger}\right)^{N_{\sigma}}|0\rangle, \quad a_{\sigma}^{\dagger} \equiv \int d^{3} r u(\vec{r}) \psi_{\sigma}^{\dagger}(\vec{r})
$$

and the statistical mixture represented by the many-boson density

$$
\sum_{\sigma}\left(a_{\sigma}^{\dagger}\right)^{N}|0\rangle \frac{N_{\sigma}}{N}\langle 0|\left(a_{\sigma}\right)^{N}
$$

lead to that same one boson density.

The condensates recently produced using all-optical confinement techniques [4] may possibly be associated with one-boson densities of type (1). This has been corroborated by applying a Stern-Gerlach magnetic field to the condensate and observing its splitting into three separate components. In general, the effect of Stern-Gerlach fields is to generate spin-position entanglement, by subjecting different spin components of the state to different boosts. One may note, moreover, that the spin components are defined with respect to a quantization axis associated to the field configuration itself, and are therefore open to manipulation. In particular, a mid-course change in the quantization axis will imply that the position amplitude associated with each of the new spin components will be a superposition of differently boosted components of the initial state. This will give interference effects at the level of the one-body density. Alternatively, a final spin selective observation scheme 
implemented in terms of a quantization axis different from that associated with the SternGerlach field will also produce one-body interference effects. Both of these schemes will be developed in what follows.

The mechanism responsible for the one-body interference can be illustrated schematically in terms of a completely polarized "travelling" one-boson amplitude

$$
\phi(\vec{r}, \sigma)=\frac{e^{i \vec{k} \cdot \vec{r}}}{\sqrt{V}} \otimes\left|\sigma_{z}=0\right\rangle
$$

If the field gradient is applied along the $x$-axis, rotation of the state changes the spin factor to $\left(\left|\sigma_{x}=1\right\rangle-\left|\sigma_{x}=-1\right\rangle\right) / \sqrt{2}$. The Stern-Gerlach boosts then change the amplitude to (up to overall position independent phase factors)

$$
\phi(\vec{r}, \sigma) \rightarrow \frac{1}{\sqrt{2 V}}\left(e^{i \vec{k} \cdot \vec{r}+i \kappa x}\left|\sigma_{x}=1\right\rangle-e^{i \vec{k} \cdot \vec{r}-i \kappa x}\left|\sigma_{x}=-1\right\rangle\right)
$$

where $\hbar \kappa$ is the momentum associated with the boosts. The spin states $\left|\sigma_{x}= \pm 1\right\rangle$ can then be re-expressed in the $z$ quantization axis as

$$
\left|\sigma_{x}= \pm 1\right\rangle=\frac{1}{2}\left(\left|\sigma_{z}=1\right\rangle \pm \sqrt{2}\left|\sigma_{z}=0\right\rangle+\left|\sigma_{z}=-1\right\rangle\right)
$$

so that the spatial density associated with the $z$-component $\sigma_{z}=1$ (say) is, after the SternGerlach entanglement

$$
\rho\left(\vec{r}, \sigma_{z}=1 ; \vec{r}, \sigma_{z}=1\right)=\frac{1}{2 V} \sin ^{2} \kappa x
$$

which shows interference fringes parallel to the z-axis. Note, in particular, that this is independent of the initial momentum $\vec{k}$ of the travelling amplitude. Eventually, an analogy may be discerned between this mechanism and that involved in Bragg diffraction experiments involving Bose-Einstein condensates [5, 6]. More specifically, the coherent superposition of amplitudes with different momenta resulting from the action of the Stern-Gerlach field after subsequent redefinition of the relevant quantization axis parallels that achieved through the action of the Bragg pulses in the first ref. [5].

We now turn to the description of the dinamics of the spinor condensate under the field gradient. We assume that when the field is applied the condensate is dilute enough so that effects of the two-body interaction are negligible. The relevant dynamics is thus contained in a second-quantized Hamiltonian of the form

$$
H=\sum_{\sigma \sigma^{\prime}} \int d^{3} r \psi_{\sigma^{\prime}}^{\dagger}(\vec{r})\left[\frac{-\hbar^{2} \nabla^{2}}{2 M} \delta_{\sigma \sigma^{\prime}}+\left\langle\sigma^{\prime}|g \vec{S} \cdot \vec{B}(\vec{r})| \sigma\right\rangle\right] \psi_{\sigma}(\vec{r})
$$

The external magnetic field $\vec{B}(\vec{r})$ will be taken to be such that there is a suitable Cartesian frame in which it has the form, assumed to be valid throughout the spatial extention of the condensate $(\sim 100 \mu m)$, 


$$
\vec{B}(\vec{r}) \rightarrow \vec{B}(x, z)=\left(B_{0}+b_{1} z\right) \hat{z}-b_{1} x \hat{x}
$$

Typical values of the constants are $B_{0} \sim 50 G$ and $b_{1} \sim 2.5 \mathrm{G} \mathrm{cm}^{-1}$.

As shown in ref [3], the dynamics can be implemented in terms of a set of semi-classical propagators associated to eigenvalues of the projection of the spin operator onto the direction of the local magnetic field. In view of the orders of magnitude given above, this direction differs negligibly from the field $z$ axis. Therefore, if this axis makes an angle $\theta$ with the quantization axis used to express the spin density, in order to apply the semi-classsical propagators one has to consider the rotated spin density

$$
\rho^{(\mathrm{s})}\left(m ; m^{\prime}\right)=\sum_{\sigma \sigma^{\prime}} d_{\sigma m}^{*}(\theta) \rho^{(\mathrm{s})}\left(\sigma ; \sigma^{\prime}\right) d_{\sigma^{\prime} m^{\prime}}(\theta)
$$

so that the complete initial density is given as

$$
\rho\left(\vec{r}, m ; \vec{r}^{\prime} m^{\prime}\right)=\rho^{(\mathrm{pos})}\left(\vec{r} ; \vec{r}^{\prime}\right) \otimes \rho^{(\mathrm{s})}\left(m ; m^{\prime}\right) .
$$

Propagation to time $t$ of each $m$ component is then performed by

$$
\left\langle x_{f}, z_{f}\left|U_{m}(t)\right| x, z\right\rangle \approx\left(\frac{M}{2 \pi i \hbar t}\right) \exp \frac{i}{\hbar} \mathcal{S}_{\text {clas }}
$$

where the classical action

$$
\mathcal{S}_{\text {clas }}=\frac{M}{2 t}\left[\left(x_{f}-x\right)^{2}+\left(z_{f}-z\right)^{2}\right]-\frac{1}{2} g m b_{1} t\left(z+z_{f}\right)-\frac{\left(g m b_{1}\right)^{2}}{24 M} t^{3}+g m B_{0} t
$$

has been evaluated using $m$-dependent trajectories in the magnetic field. Note that $m$ is a constant of motion along the trajectories due to the special choice of reference frame 3]. The evaluation of the above expression involved neglecting terms of the order $\left(b_{1} \Delta x / B_{0}\right)^{2}$, with $\Delta x$ of the order of the propagation range. Since the evolution in the $y$ direction is free, it has been ommited from the above expressions. The propagated density at time $t$ is therefore given by

$$
\rho\left(\vec{r}, m ; \vec{r}^{\prime} m^{\prime}, t\right)=\int d^{3} r_{1} \int d^{3} r_{2}\left\langle\vec{r}\left|U_{m}(t)\right| \vec{r}_{1}\right\rangle \rho\left(\vec{r}_{1}, m ; \vec{r}_{2}, m^{\prime}\right)\left\langle\vec{r}_{2}\left|U_{m^{\prime}}^{\dagger}(t)\right| \vec{r}^{\prime}\right\rangle .
$$

The spin inclusive observed spatial density after propagation in the Stern-Gerlach field is then given by the trace

$$
\rho^{(\mathrm{obs})}(\vec{r}, t)=\sum_{m} \rho(\vec{r}, m ; \vec{r}, m, t)
$$

In the case of an initial spin density which is diagonal in the magnetic field frame with weights $3 / 5: 1 / 5: 1 / 5$ for the components $-1,0,+1$ and an initial gaussian spatial distribution one obtains the result shown in fig. 1(a), for $t=15 \mathrm{~ms}$ and field gradient somewhat lower than 
the typical value quoted above. The resulting distribution closely resembles that given in

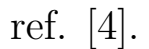

We next consider a mid-course change of the Stern-Gerlach field. After a first stage described by the above expressions and lasting for a time $t_{1}$ a sudden rotation by an angle $\theta_{B}$ of $\vec{B}(x, z)$ is performed around the $y$ axis. Strictly speaking, it is not important that the field values are maintained, so that the rotation can be achieved e.g. by superimposing an additional external field. The initial condition for the second stage of the evolution will be then, in the appropriate field frame

$$
\rho^{(2)}\left(\vec{r}, m ; \vec{r}^{\prime} m^{\prime}, t_{1}\right)=\sum_{m_{1} m_{2}} d_{m_{1} m}^{*}\left(\theta_{B}\right) \rho\left(\vec{r}, m_{1} ; \vec{r}^{\prime} m_{2}, t_{1}\right) d_{m_{2} m^{\prime}}\left(\theta_{B}\right)
$$

The propagation to $t>t_{1}$ is handled analogously to the first stage, in terms of a propagator $\tilde{U}_{m}\left(t-t_{1}\right)$, leading to a final density

$$
\rho^{(2)}\left(\vec{r}, m ; \vec{r}^{\prime} m^{\prime}, t\right)=\int d^{3} r_{1} \int d^{3} r_{2}\left\langle\vec{r}\left|\tilde{U}_{m}\left(t-t_{1}\right)\right| \vec{r}_{1}\right\rangle \rho^{(2)}\left(\vec{r}_{1}, m ; \vec{r}_{2}, m^{\prime}, t_{1}\right)\left\langle\vec{r}_{2}\left|\tilde{U}_{m^{\prime}}^{\dagger}\left(t-t_{1}\right)\right| \vec{r}^{\prime}\right\rangle .
$$

The corresponding spin-inclusive final spatial density is now

$$
\begin{aligned}
\rho^{(\mathrm{inc})}(\vec{r}, t) & =\sum_{m} \rho^{(2)}(\vec{r}, m ; \vec{r}, m, t)= \\
& =\sum_{m} \sum_{m_{1} m_{2}} \int d^{3} r_{1} \int d^{3} r_{2}\left\langle\vec{r}\left|\mathcal{U}_{m_{m}}(t)\right| \vec{r}_{1}\right\rangle \rho\left(\vec{r}_{1}, m_{1} ; \vec{r}_{2}, m_{2}\right)\left\langle\vec{r}_{2}\left|\mathcal{U}_{m_{2} m}^{\dagger}(t)\right| \vec{r}\right\rangle
\end{aligned}
$$

where

$$
\left\langle\vec{r}\left|\mathcal{U}_{m m^{\prime}}(t)\right| \vec{r}^{\prime}\right\rangle=\int d^{3} r_{1}\left\langle\vec{r}\left|\tilde{U}_{m}\left(t-t_{1}\right)\right| \vec{r}_{1}\right\rangle d_{m^{\prime} m}^{*}\left(\theta_{B}\right)\left\langle\vec{r}_{1}\left|U_{m^{\prime}}\left(t_{1}\right)\right| \vec{r}^{\prime}\right\rangle
$$

is the effective, spin non-diagonal propagator from time zero to time $t$. Note that, during the first stage, different $m$ components are subjected to different boosts. After the rotation $\theta_{B}$, the new $m$ components will in general be associated with coherent superpositions of differently boosted spatial amplitudes, leading to interferece patterns in the observed spin inclusive one-boson density, as can be seen in fig. 1. This interference will be absent in the special case in which the spin density is diagonal in the reference frame defined by the field acting in the first stage, due to the incoherent nature of the differently boosted components.

Alternatively, we may let the system evolve as in the first stage for a time $t$, and then consider the resulting one-boson density spin-selected along a quantization axis differing from the field $z$ axis by a rotation $\theta_{\text {sel }}$ around the $y$ axis. In this case the spatial one-boson density selected for the component $m$ of the spin in the last frame will be given by

$$
\rho^{(\mathrm{sel})}(\vec{r}, t)=\sum_{m_{1} m_{2}} d_{m_{1} m}^{*}\left(\theta_{\mathrm{sel}}\right) \rho\left(\vec{r}, m_{1} ; \vec{r}, m_{2}, t\right) d_{m_{2} m}\left(\theta_{\mathrm{sel}}\right) .
$$

which will also show interference effects for the same general reasons discussed in the previous case. In particular, in the special case in which the spin density is diagonal in the reference 
frame defined by the field, no interference occurs in the one-boson density. An example of this case is shown in fig. 2 .

The initial (gaussian) spatial amplitudes leading to the patterns shown in Figs. 1 and 2 have been taken as real, and therefore the initial velocity field vanishes. The regime in which the two-body interaction effects becomes negligible is in general reached after release of the mean-field energy, which generates a non-vanishing velocity field. The argument given above in order to illustrate the one-boson interference mechanism indicates that this initial velocity field does not change the interference pattern generated by the Stern-Gerlach boosts. This has in fact been verified in the calculations, by starting from narrower gaussians and letting them spread freely for the appropriate time before applying the magnetic field.

In both cases, the typical fringe spacing is determined by the de Broglie wavelength associated with the relative velocities of the interfering amplitudes. This depends both on the value of the magnetic field gradient and on the duration of the first stage (in the first case above) or of the exposure time to the field (in the second case). For the $F=1$ states of ${ }^{85} \mathrm{Rb}$, fringes of the order of several microns correspond to times of the order of $1 \mathrm{~ms}$ or

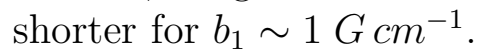

It should be stressed that the above analysis has been carried out at the level of the one-boson density. To the extent that laboratory observation is sensitive to many-boson correlations [7], further interference effects are to be expected. Notably, in the case of the pure many-boson initial state (2) the Monte Carlo procedure of ref. [7] will generate an interference pattern in the extended $\vec{r}, \sigma$ space even when the initial Stern-Gerlach boosts are applied along the $z$-axis, which is however invisible at the one-boson level. No many-body interferences occur, under these conditions, in the case of the mixed many-boson state (3), due to the incoherece of the different spin components. If, however, the initial Stern-Gerlach boost is applied along a direction different from the $x$-axis, each one of the incoherent, completely polarized spin components of the mixed many-boson state will generate an interference pattern which seeps down to the one-body level. The restriction to the one-body level appears thus as a "filter" on the richer domain of many-body effects.

Acknowledgement. S. C.-B. has been partially supported by the Spanish CICyT, Project No. PB98-1111 and by the Pró-Reitoria de Pesquisa of the Universidade de São Paulo, and M.C.N. has been partially supported by by the Fundação de Amparo à Pesquisa do Estado de São Paulo (FAPESP) and by the Conselho Nacional de Desenvolvimento Científico e Tecnológico (CNPq).

\section{References}

[1] W. Gerlach and O. Stern, Z. Phys. 8, 110 (1922); 9, 349 (1922).

[2] In this context, the coherence properties of the spatially separated components of the wave function have been extensively discussed in connection with the "reconstruction" of the initial spin state, see Z. Hradil, J. Summhammer, G. Badurek and H. Rauch, Phys. Rev. A62, 014101 (2000) and references therein. 
[3] S. Cruz-Barrios and J. Gómez-Camacho, Phys. Rev. A63, 012101 (2001).

[4] M. D. Barrett, A. J. Sauer and M. S. Chapman, Phys. Rev. Lett. 87, 010404 (2001).

[5] M Kozuma, L. Deng, E. W. Hagley, J. Wen, L. Lutwak, K. Helmerson, S. L. Rolston and W. D. Phillips, Phys. Rev. Lett. 82, 871 (1999); M. Kozuma, Y. Suzuki, Y. Torii, T. Sugiura, T. Kuga E. W. Hagley and L. Deng, Science 286, 2309 (1999); J. P. E. Simsarian, J. Denschlag, M. Edwards, C. W. Clark, L. Dend, E. W. Hagley, K. Helmerson, S. L. Holston and W. D. Phillips, Phys. Rev. Lett. 85, 2040 (2000).

[6] J. Stenger, S. Inouye, A. P. Chikkatur, D. M. Stamper-Kurn, D. E. Pritcahrd and W. Ketterle, Phys. Rev. Lett. 82, 4569 (1999); S. Inouye, R. F. Lw, S. Gupta, T. Pfau, A. Grlitz, T. L. Gustavson, D. E. Pritcahrd and W. Ketterle, Phys. Rev. Lett. 85, 4225 (2000).

[7] J. Javanainen and S. M. Yoo, Phys. Rev. Lett. 76, 161 (1996). 
Figure 1: Contour plots (sizes given in $\mu m$ ) of the spin inclusive one-boson density (4) integrated over $y$ for the shown values of $t_{1}$ and $t$. The initial one-boson spin density is diagonal with eigenvalue ratios $3: 1: 1$ for spin $z$-components $-1,0,+1$ respectively. The initial gaussian amplitude has $b_{x}=25 \mu \mathrm{m}$ and $b_{y, z}=$ $15 \mu \mathrm{m}$. The angles $\theta$ and $\theta_{B}$ are $\pi / 2$ and $-\pi / 2$ respectively. Magnetic field parameters: $B_{0}=50 \mathrm{G}$ and $b_{1}=1.0 \mathrm{Gcm}^{-1}$. Note that in (c) interference fringes are too narow for accurate representation.
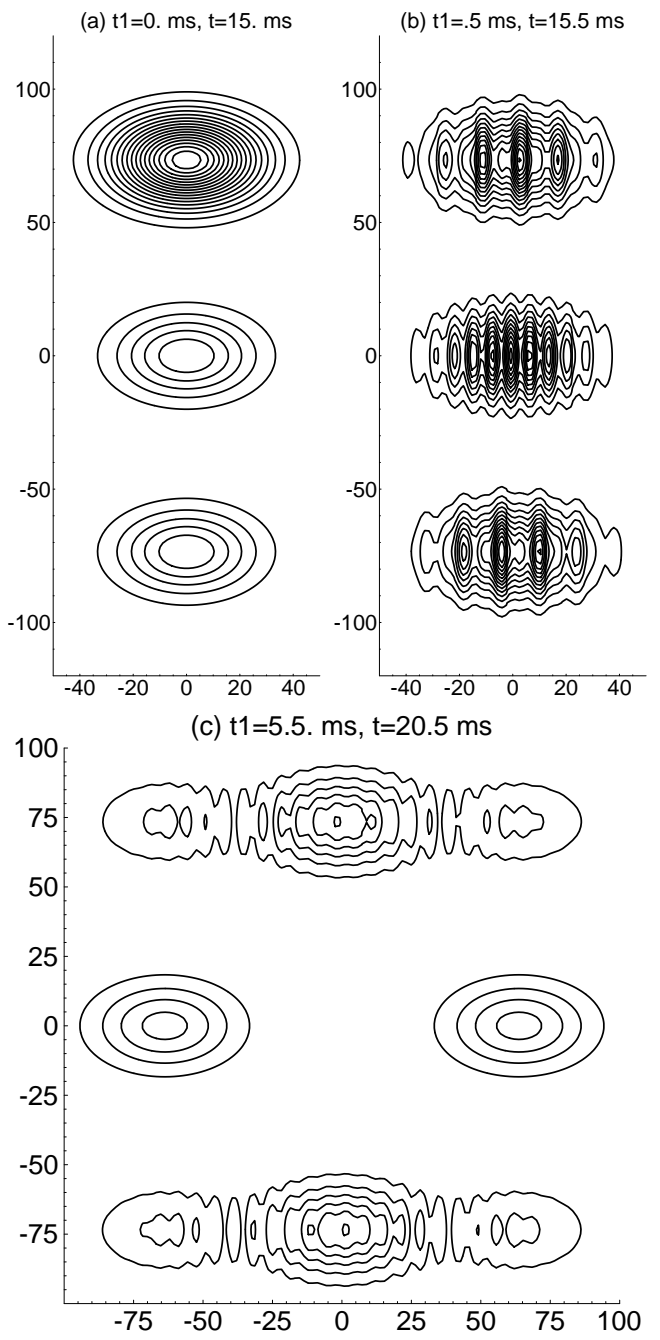
Figure 2: Contour plots (sizes given in $\mu m$ ) of the $m=+1$ spin selected oneboson density (5) integrated over $y$ for the shown values of $t$. The initial one-boson spin density is a pure $s_{z}=-1$ state. The angles $\theta$ and $\theta_{\text {sel }}$ are $\pi / 4$ and $\pi / 2$ respectively. Gaussian and field parameters are the same as in Fig. 1. Note that in (b) interference fringes are too narow for accurate representation.

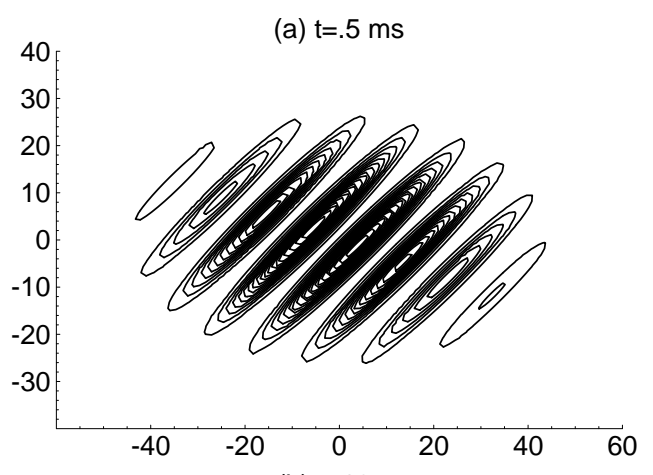

(b) $\mathrm{t}=12 \mathrm{~ms}$

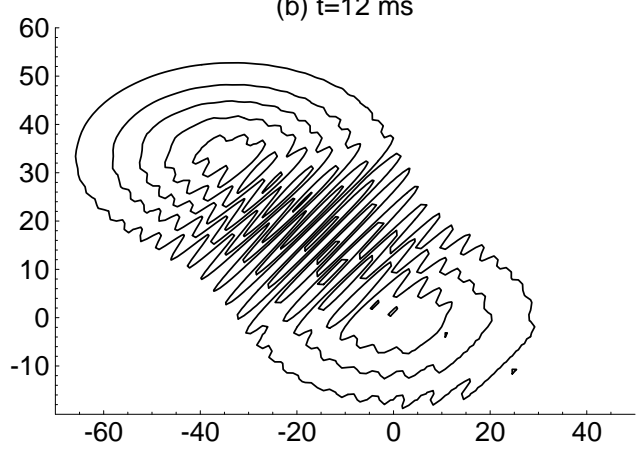

